\title{
Author Correction: The effect of LRRK2 loss-of-function variants in humans
}

Nicola Whiffin (1), Irina M. Armean (1), Aaron Kleinman, Jamie L. Marshall(1), Eric V. Minikel, Julia K. Goodrich,

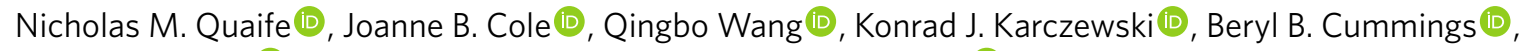
Laurent Francioli@, Kristen Laricchia, Anna Guan, Babak Alipanahi @i, Peter Morrison, Marco A. S. Baptista, Kalpana M. Merchant, Genome Aggregation Database Production Team, Genome Aggregation Database Consortium*,

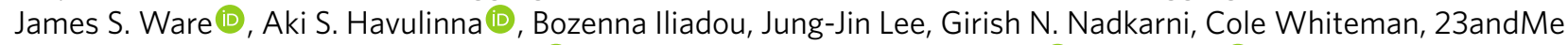
Research Team^, Mark Daly, Tõnu Esko (1), Christina Hultman, Ruth J. F. Loos (D), Lili Milani (D), Aarno Palotie, Carlos Pato, Michele Pato, Danish Saleheen (1D, Patrick F. Sullivan, Jessica Alföldi (D, Paul Cannon and Daniel G. MacArthur (D)

Correction to: Nature Medicine https://doi.org/10.1038/s41591-020-0893-5, published online 27 May 2020.

In the version of this article initially published, an author (Marquis P. Vawter) was missing from the 'Genome Aggregation Database Consortium' list. The correct listing is “...Erkki Vartiainen ${ }^{92}$, Marquis P. Vawter ${ }^{149}$, James S. Ware ${ }^{1,2,3}$...”; the affiliation is “149 Department of Psychiatry \& Human Behavior, University of California Irvine, Irvine, CA, USA.” (with subsequent affiliations renumbered accordingly). Also, funding information for author Laurent Francioli was not provided. The correct text is as follows: “...NIHR Imperial Biomedical Research Centre. L.F. was supported by the Swiss National Science Foundation (Advanced Postdoc. Mobility 177853). T.E is supported by.... The errors have been corrected in the HTML and PDF versions of the article.

${ }^{\star}$ Lists of authors and their affiliations appear online.

Published online: 22 January 2021

https://doi.org/10.1038/s41591-020-01185-6

(c) The Author(s), under exclusive licence to Springer Nature America, Inc. 2021

\section{Author Correction: Multimodel preclinical platform predicts clinical response of melanoma to immunotherapy}

Eva Pérez-Guijarro (1), Howard H. Yang, Romina E. Araya, Rajaa El Meskini, Helen T. Michael, Suman Kumar Vodnala, Kerrie L. Marie, Cari Smith, Sung Chin, Khiem C. Lam, Andres Thorkelsson, Anthony J. lacovelli, Alan Kulaga, Anyen Fon, Aleksandra M. Michalowski, Willy Hugo, Roger S. Lo, Nicholas P. Restifo, Shyam K. Sharan (1D, Terry Van Dyke,

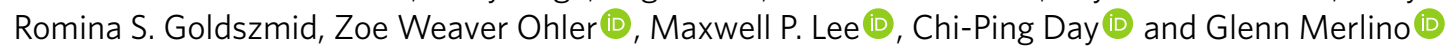

Correction to: Nature Medicine https://doi.org/10.1038/s41591-020-0818-3, published online 13 April 2020.

In the version of this article initially published, the designation $\mathrm{H} 2-\mathrm{Kd}$ (or $\mathrm{H}-2 \mathrm{Kd}$ ) is incorrect in the third sentence of the first paragraph of the third subsection of Results ('Antigen-presentation pathway is functional in the four melanoma models') and in the second sentence of first paragraph of the sixth subsection of Methods ('Antigen presentation, T cell activation assays and flow cytometry'), as well as in Supplementary Fig. $2 \mathrm{~b}$ and its legend. The correct designation is $\mathrm{H} 2-\mathrm{Kb}$. The error has been corrected in the HTML and PDF versions of the article.

Published online: 29 January 2021

https://doi.org/10.1038/s41591-021-01252-6

(c) The Author(s), under exclusive licence to Springer Nature America, Inc. 2021 